\title{
Persistence of platelet thrombus formation in arterioles of mice lacking both von Willebrand factor and fibrinogen
}

\author{
Heyu Ni, ${ }^{1,2}$ Cécile V. Denis, ${ }^{1,2}$ Sangeetha Subbarao, ${ }^{1}$ Jay L. Degen, ${ }^{3}$ Thomas N. Sato, ${ }^{4}$ \\ Richard O. Hynes, ${ }^{5}$ and Denisa D. Wagner ${ }^{1,2}$
}

${ }^{1}$ The Center for Blood Research, and
${ }^{2}$ Department of Pathology, Harvard Medical School, Boston, Massachusetts, USA
${ }^{3}$ Children's Hospital Research Foundation and University of Cincinnati, Cincinnati, Ohio, USA
${ }^{4}$ The University of Texas Southwestern Medical Center at Dallas, Dallas, Texas, USA
${ }^{5}$ Howard Hughes Medical Institute and Center for Cancer Research, Department of Biology,
Massachusetts Institute of Technology, Cambridge, Massachusetts, USA

Address correspondence to: Denisa D. Wagner, The Center for Blood Research, 800 Huntington Avenue, Boston, Massachusetts 02115, USA. Phone: (617) 278-3344; Fax: (617) 278-3368; E-mail: wagner@cbr.med.harvard.edu.

Received for publication March 17, 2000, and accepted in revised form June 22, 2000.

\begin{abstract}
We used intravital microscopy to observe the formation of platelet plugs in ferric chloride-injured arterioles of live mice. With this model, we evaluated thrombus growth in mice lacking von Willebrand factor (vWF) and fibrinogen (Fg), the two key ligands known to mediate platelet adhesion and aggregation. In $v \mathrm{WF}^{-/-}$mice, despite the presence of arterial shear, delayed platelet adhesion occurred and stable thrombi formed. In many mice, a persisting high-shear channel never occluded. Abundant thrombi formed in $\mathrm{Fg}^{-/-}$mice, but they detached from the subendothelium, which ultimately caused downstream occlusion in all cases. Surprisingly, mice deficient in both vWF and Fg successfully formed thrombi with properties characteristic of both mutations, leading to vessel occlusion in the majority of vessels. Platelets of these doubly deficient mice specifically accumulated fibronectin in their $\alpha$-granules, suggesting that fibronectin could be the ligand supporting the platelet aggregation.
\end{abstract}

J. Clin. Invest. 106:385-392 (2000).

\section{Introduction}

Platelet adhesion and aggregation at the site of vascular injury are key events leading to the formation of a platelet plug and subsequent arrest of bleeding. The two principal ligands known to mediate platelet adhesion and aggregation are von Willebrand factor (vWF) and fibrinogen $(\mathrm{Fg})$, whose importance is underlined by the bleeding disorders associated with their respective deficiencies, i.e., von Willebrand's disease (vWd) and afibrinogenemia $(1,2)$. Symptomatically affecting as much as $0.01-0.1 \%$ of the world's population, $\mathrm{vWd}$ is the most common inherited bleeding disorder and is characterized by frequent mucocutaneous bleeding and prolonged bleeding times from trivial wounds or minor surgery (3). Congenital afibrinogenemia, described in approximately 150 families, also leads to a bleeding diathesis that can be fatal in approximately one-third of such patients (4). Another aspect of vWF and Fg is their likely involvement in thrombosis. Thrombosis in coronary or cerebral arteries is the major cause of morbidity and mortality worldwide. Thus, understanding the specific roles of vWF and Fg in hemostasis and thrombosis is important to prevent and treat myriad hemorrhagic and cardiovascular disorders.

The roles of vWF and Fg in platelet adhesion and aggregation have been extensively studied in vitro. At high shear rate, conditions encountered in the arteries and in the microcirculation, vWF has been shown to promote platelet adhesion to the subendothelium (5,
6). To perform this function, subendothelial-bound vWF binds to its platelet receptor glycoprotein (GP) Ib, establishing a transient bond that can slow down the platelets and lead to activation of platelet $\alpha \operatorname{IIb} \beta 3$ (integrin GPIIb/IIIa) $(7,8)$. After that first adhesion step, different receptor-ligand interactions synergistically promote stable platelet adhesion (9). For example, platelet integrin $\alpha 2 \beta 1$ is involved in the adhesion step through its interaction with collagens $(9,10)$, and $\alpha \operatorname{IIb} \beta 3$ facilitates platelet adhesion and spreading (9, 11). Furthermore, $\alpha \operatorname{IIb} \beta 3$ also mediates subsequent platelet-platelet interactions through its multivalent ligands, Fg and/or vWF (12-16). In contrast to vWF, which is thought to be the important ligand at high shear rates, at low shear rates in veins Fg is thought to be the main player in both platelet adhesion and aggregation through its interaction with $\alpha \operatorname{IIb} \beta 3(13,17)$.

Most studies of platelet thrombus formation have used in vitro systems with purified components or anticoagulated blood. However, the precise roles that vWF and Fg play in the dynamic environment of an in vivo injury, where all the natural basement membrane and blood constituents are present, remain to be rigorously examined. In the present study, we took advantage of the availability of gene-knockout mice with deficiencies in vWF (18) and Fg (19) to explore the role of these ligands in platelet plug formation in our recently developed intravital microscopy/thrombosis model (18). In this model, vascular injury is induced by superfusion of 
ferric chloride on an arteriole of mouse mesentery. This injury triggers the generation of free radicals leading to the disruption and denudation of endothelium (20). The deposition of fluorescently labeled platelets at the injured site is then observed and recorded in real time.

\section{Methods}

Mice. C57BL/6J/129Sv $v \mathrm{WF}^{-/}$and $\mathrm{Fg}^{-/-}$mice were described $(18,19)$. To minimize the influence of straindependent genetic differences, we produced the $\nu \mathrm{WF}^{+/+}$ and $v \mathrm{WF}^{-/-}$mice used in this study from $v \mathrm{WF}^{+/-}$crosses and $\mathrm{Fg}^{+/+}$and $\mathrm{Fg}^{-/-}$from $\mathrm{Fg}^{+/-}$crosses. The homozygous and corresponding wild-type lines were then bred separately for a few generations, avoiding brother-sister crosses. This could have generated some minor differences in background composition of these mice. However, we have compared the thrombus formation in the two sets of wildtype mice $\left(\mathrm{Fg}^{+/+}\right.$and $\left.v \mathrm{WF}^{+/+}\right)$and found them indistinguishable, with no significant difference in occlusion time $(P=0.42)$. To generate $\nu \mathrm{WF} / \mathrm{Fg}^{-1-}$ mice, $\nu \mathrm{WF}^{-/-}$females and $\mathrm{Fg}^{-/-}$males were bred. $\mathrm{Fg}^{+/-}$and $\nu \mathrm{WF}^{+/-} \mathrm{F} 1$ mice were crossed to obtain double-knockout male mice and $v \mathrm{WF}^{-1-}$, $\mathrm{Fg}^{+/-}$females. These mice were used to generate doubleknockout pups for experiments because $\mathrm{Fg}^{-1}$ female mice succumb to uterine bleeding during gestation (19). A mix of $\mathrm{Fg}^{+/+}$and $\nu \mathrm{WF}^{+/+}$mice was used as wild type.

Generation of Tie2-GFP transgenic mice and time course of arteriolar injury after $\mathrm{FeCl}_{3}$ treatment. Enhanced and redshifted green fluorescent protein (GFP) was specifically expressed in vascular endothelial cells under the control of TIE2 promoter and enhancer elements (21). A fulllength NotI-NotI GFP cDNA fragment from pGreen Lantern-1 (Life Technologies, Gaithersburg, Maryland, USA) was flanked by the 2-kb HindIII-HindIII promoter fragment upstream and the SV40 polyA signal sequences and the 10-kb NaeIII-NotI enhancer fragment downstream. The TIE2 promoter/enhancer GFP transgenic construct was purified and injected into FVB oocytes (21). Genotyping of the mice was performed with a forward primer in the TIE2 promoter and a reverse primer in the GFP cDNA. The PCR protocol and request procedure for the transgenic mice are available from http://cbi.swmed.edu/ryburn/sato.

Abdominal incisions were performed on the GFP mice, arteriolar injury was induced by $\mathrm{FeCl}_{3}$, and fluorescence of mesenteric arterioles $(80-120 \mu \mathrm{m}$ diameter) was visualized and recorded (18). Twenty-eight arterioles were randomly divided into untreated and $\mathrm{FeCl}_{3}$-treated groups. To minimize bleaching by ultraviolet (UV) light, the fluorescence image was recorded for 3 seconds before $\mathrm{FeCl}_{3}$ treatment and then at the end of each minute after $\mathrm{FeCl}_{3}$ treatment. The same treatment was performed in control vessels. The fluorescence intensities were ranked from grade 5 (most intense) to 0 (no fluorescence), and statistical analysis was performed using the Mann-Whitney $U$ test.

Immunohistochemical staining. $\mathrm{FeCl}_{3}$-treated and untreated wild-type mesenteric arterioles were fixed for $10 \mathrm{~min}$ utes with $10 \%$ buffered formalin (Fisher Scientific, Pitts- burgh, Pennsylvania, USA), washed with PBS, and frozen in OCT (Sakura Finetek, Torrance, California, USA). Cryostat sections were treated with cold acetone for 5 minutes. Endogenous peroxidase was quenched by $3 \%$ $\mathrm{H}_{2} \mathrm{O}_{2}$ for 10 minutes. Sections were blocked by normal serum and incubated for 60 minutes with 1:100 diluted rabbit anti-human vWF polyclonal Ab (ASSERA; Diagnostica Stago, Asnieres-sur-Seine, France). Goat anti-rabbit biotinylated $\mathrm{Ab}$, streptavidin-horseradish peroxidase conjugate and substrate (aminoethylcarbazole chromogen) were used according to the manufacturer's instructions (Zymed Laboratories Inc., South San Francisco, California, USA). Sections were counterstained with hematoxylin (Zymed Laboratories Inc.). For PECAM-1 (CD31) staining, MEC 13.3, a rat mAb (PharMingen, San Diego, California, USA), was used at a ratio of $1: 20$.

Immunofluorescence staining. Gel-filtered platelets were smeared and air dried on microscope slides (Fisher Scientific). Smears were fixed in 3.7\% formaldehyde in PBS at room temperature for 20 minutes and permeabilized in $10 \%$ Triton-X 100 in PBS buffer for 15 minutes. The platelets were incubated first with 1:200 diluted rabbit anti-mouse fibronectin (22) or thrombospondin-1 (kindly provided by Deane F. Mosher, University of Wisconsin, Madison, Wisconsin, USA), or vitronectin (kindly provided by David Loskutoff, Scripps Research Institute, La Jolla, California, USA) at $37^{\circ} \mathrm{C}$ for $30 \mathrm{~min}$ utes, then with 1:500-diluted FITC-labeled goat antirabbit IgG (Cappel-ICN Pharmaceuticals, Biochemical Division, Aurora, Ohio, USA) at $37^{\circ} \mathrm{C}$ for 30 minutes.

Flow cytometry. Blood samples from six mice of each genotype were pooled, and platelets were isolated (18). For labeling $\alpha \operatorname{IIb} \beta 3$, platelets were incubated 30 minutes at room temperature with 1:200-diluted rat mAb D9 to mouse $\alpha \mathrm{IIb} \beta 3$ (kindly provided by A.K. Ng, University of Southern Maine, Portland, Maine, USA) and FITC-goat anti-rat IgG (Cappel-Organon Teknika Corp., Durham, North Carolina, USA) 1:500 for 30 minutes. Ten thousand platelets were analyzed using a FACScalibur (Becton Dickinson, San Jose, California, USA). $\beta 1$ integrins were stained with 1:200-diluted FITC-hamster anti-rat CD29 antibody Ha2/5 (PharMingen), which cross-reacts with mouse CD29 for 30 minutes.

Western blot analysis. Blood samples from six mice of each genotype were pooled and platelets were isolated on a Sepharose $2 \mathrm{~B}$ column. Platelets $\left(10^{8}\right)$ were lysed in $45 \mu \mathrm{L}$ lysis buffer (0.1 M Tris-Cl, pH 8.3, 0.2\% SDS, $10 \%$ glycerol, 4 mM EDTA, 2 mM $N$-methylmaleimide, 2 $\mathrm{mM}$ iodoacetic acid, $4 \mathrm{mM}$ PMSF), $5 \mu \mathrm{L} 1 \mathrm{M}$ DTT was added, and the samples boiled for 5 minutes. Proteins were separated in 6\% SDS-PAGE gel and transferred to Immobilon-P PVDF membrane (Millipore Corp., Bedford, Massachusetts, USA). This membrane was blocked with 5\% nonfat milk TBST (20 mM Tris-Cl, pH 7.6, 137 $\mathrm{mM} \mathrm{NaCl}, 0.1 \%$ Tween 20), then incubated with 1:2,000-rabbit anti-mouse fibronectin (22), or rabbit anti-mouse thrombospondin-1, or with 1:1,000-rabbit anti-mouse vitronectin (antibodies as above) at room 

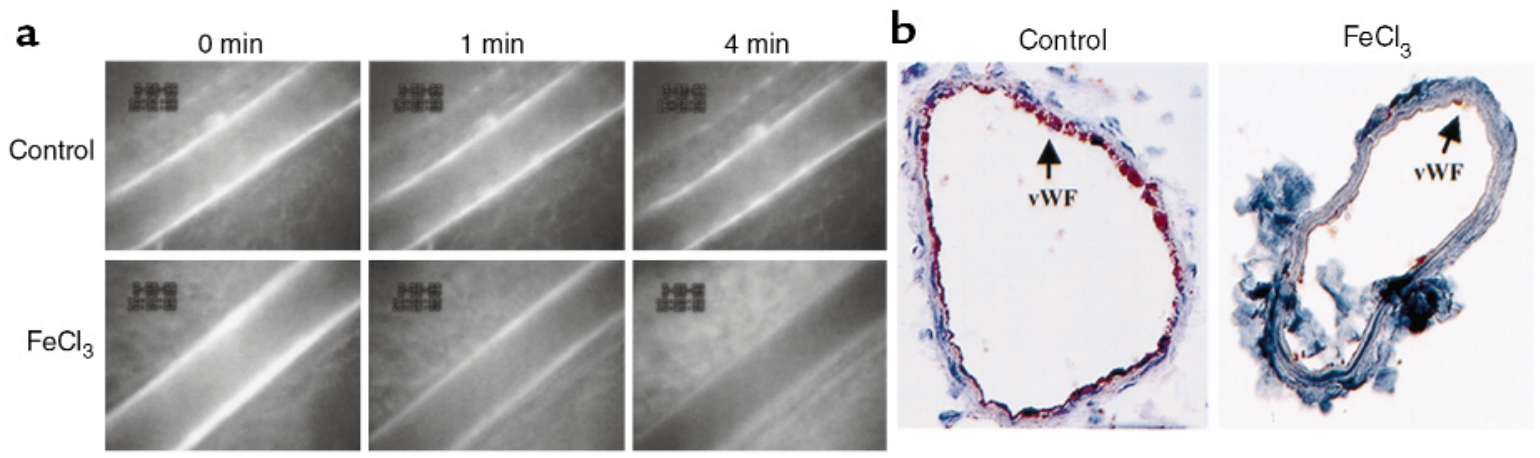

Figure 1

Arteriolar injury and denudation of endothelium induced by $\mathrm{FeCl}_{3}$. (a) Progression of $\mathrm{FeCl}_{3}$-induced injury. Fluorescence intensity of endothelium of mesenteric arterioles ( $n=14$ /group) from GFP-transgenic mice was blindly ranked from 5 to 0 . $\mathrm{FeCl}_{3}$-treated or untreated arterioles were then compared by Mann-Whitney $U$ test. Before $\mathrm{FeCl}_{3}$ treatment $(0 \mathrm{~min})$, all arterioles had a similar intensity $(\sim 5)$. Significant differences were found after 1 minute of $\mathrm{FeCl}_{3}$ exposure $(P=0.0006)$. After 2 minutes (not shown), the intensity in control vessels decreased only slightly (mean $=4.1$ ), whereas in $\mathrm{FeCl}_{3}$-treated vessels, fluorescence was greatly decreased (mean $\left.=1.4 ; P=0.0001\right)$. After 4 minutes, the fluorescence intensity in the control group was still high (mean = 3.4), but in the $\mathrm{FeCl}_{3}$ group was almost undetectable (mean $=0.4 ; P<$ $0.0001)$. Representative photographs of one vessel from each group are shown. (b) Denudation of endothelium by $\mathrm{FeCl}_{3}$. Control $(\sim 130$ $\mu \mathrm{m}$ diameter) and $\mathrm{FeCl}_{3}$-treated ( $\sim 100 \mu \mathrm{m}$ diameter $)$ sections of mesenteric arterioles from wild-type mice were stained with rabbit antihuman vWF polyclonal Ab, which cross-reacts with mouse vWF. Endothelial cells in the control arteriole were heavily stained, whereas only a few areas were stained with this $\mathrm{Ab}$ (arrow) after 5-minute $\mathrm{FeCl}_{3}$ treatment. These areas may be remaining endothelial cells or small thrombi. The brown ring at the inner side of the injured vessel wall may result from plasma vWF deposition or from subendothelial vWF.

temperature for 2 hours. Membrane was then incubated with 1:100 ${ }^{125}$ I-labeled goat anti-rabbit $\operatorname{IgG}(1$ $\mu \mathrm{Ci} / \mathrm{mL}$; NEN Life Science Products Inc., Boston, Massachusetts, USA), revealed with a PhosphorImager (Molecular Dynamics Storm 860) and analyzed by IMAGEQUANT (Molecular Dynamics Inc., Sunnyvale, California, USA). To compare the plasma levels of fibronectin, vitronectin, and thrombospondin-1 in wildtype and $v \mathrm{WF} / \mathrm{Fg}^{-1-}$ mice, proteins of individual plasma samples (eight mice per each genotype) were separated by $7 \%$ SDS-PAGE gels, and blots were developed by the chemiluminescence method (ECL; Amersham Pharmacia Biotech Inc., Piscataway, New Jersey, USA).

In vivo thrombosis model. As described previously (18), male mice (3.5-4.5 weeks old) were injected with the fluorescently labeled platelets $\left(5 \times 10^{6} / \mathrm{g}\right)$ of matching genotype in the lateral tail vein. The mice were anesthetized, and the mesentery was exteriorized through a midline abdominal incision. Arterioles were visualized with a Zeiss Axiovert 135-inverted microscope (objective 32×, 0.4NA; Zeiss, Oberkochen, Germany) and recorded on videotape. Vessels were monitored for 40 minutes after $\mathrm{FeCl}_{3}$ treatment or until full occlusion occurred (blood flow stopped) and lasted for more than 10 seconds.

Statistical analysis. Data are presented as mean plus or minus SEM. Statistical significance was assessed by $t$ test or by Mann-Whitney $U$ test and $X^{2}$ test as indicated.

\section{Results}

Characterization of the arteriolar injury in the intravital microscopy thrombosis model. Induction of vascular injury and thrombus formation with $\mathrm{FeCl}_{3}$ has been reported in a variety of species and vascular sites (18, $20,23,24)$. To examine the time course of $\mathrm{FeCl}_{3}$ effect on the endothelium of the mesenteric arteriole, we employed transgenic mice in which the GFP was specifically expressed in endothelial cells. We found that fluorescence intensity in mesenteric arterioles markedly decreased after a 1-2 minutes of $\mathrm{FeCl}_{3}$ application in comparison with control vessels (Figure 1a). This decrease shows that $\mathrm{FeCl}_{3}$ penetrated the endothelium rapidly and suggests that endothelial injury occurred shortly after $\mathrm{FeCl}_{3}$ application. However, the loss of fluorescence intensity does not distinguish between the denudation of endothelium and the quenching of fluorescence by direct $\mathrm{FeCl}_{3}$-GFP interaction inside the cell at the onset of lysis. We therefore stained endothelia of mesenteric arterioles with anti-PECAM-1 and anti-vWF antibodies. The presence of endothelial cells containing PECAM-1 (not shown) or vWF, clustered in Weibel-Palade bodies (25), was dramatically decreased after 5 minutes of $\mathrm{FeCl}_{3}$ treatment (Figure 1b). Thus, $\mathrm{FeCl}_{3}$ induced removal of endothelia, which led to exposure of subendothelial matrix. The shear rate $\left(\sim 1300 \mathrm{~s}^{-1}\right)$ and diameter of the arterioles $(\sim 100 \mu \mathrm{m})$ were similar for all genotypes studied. After $\mathrm{FeCl}_{3}$ application, shear rate increased about $30 \%$ in the first $10-20$ seconds and then returned to the untreated level within 40 seconds. After this, no significant change in shear rate was observed during the experimental period. We would like to add that this is a mouse thrombosis model, which may not be directly comparable to humans. Mouse platelets are smaller and more numerous than those in humans (26), and thus the shear effects could be different.

Delayed thrombus initiation and defective vessel occlusion in $v$ WF-knockout mice. In the wild-type animals, unstable platelet interactions with the vessel wall started around 20-30 seconds after topical application of 


\section{Table 1}

The effect of vWF and Fg deficiency on early platelet-vessel wall interaction and thrombus formation

$\begin{array}{lcc}\text { Genotype } & \begin{array}{c}\text { Platelet deposition } \\ \text { (3-5 min after injury) } \\ \text { Number of platelets } / \text { min }\end{array} & \begin{array}{c}\text { Time required for formation } \\ \text { of the first thrombus } \\ (>20 \mu \mathrm{m})(\mathrm{min})\end{array} \\ \text { Wild-type } & 159.6 \pm 18.9 & 4.3 \pm 0.9 \\ \mathrm{vWF} F^{--} & 32.2 \pm 19.0 & 9.4 \pm 1.6 \\ \mathrm{Fg}^{-/-} & 138.4 \pm 19.2 & 5.5 \pm 0.9 \\ \mathrm{vWF} / \mathrm{Fg}^{-/-} & 50.0 \pm 21.3 & 13.2 \pm 2.9\end{array}$

The numbers of fluorescently labeled platelets deposited on vessel walls between $3-5$ minutes after injury were counted over a $410-\mu \mathrm{m}$ length of vessel. Significant differences were observed between $v W F^{-1-}$ and wild-type $(P=0.0001)$ or $v W F / F^{-/-}$and wild-type $(P=0.0009)$ mice. No significant difference in platelet-vessel wall interaction was found between $\mathrm{Fg}^{-1}$ - and wild-type $(P=0.45)$ or $v W F^{-1-}$ and $v W F / \mathrm{Fg}^{-1-}$ mice $(P=0.54)$. The time required for the appearance of the first thrombus after injury was measured. Significant differences were found between $v W F^{-/-}$and wild-type $(P=0.008)$ or $v W F / F^{-1-}$ and wild-type $(P=0.006)$ but no significant difference was found between $\mathrm{Fg}^{-1-}$ and wild-type $(P=0.33)$ or $v W F^{-1-}$ and $v W F / F^{-1-}$ mice $(P=0.28) \cdot n=10-17$.

$\mathrm{FeCl}_{3}$. Two to 3 minutes later, numerous fluorescent adherent platelets were found (Figure 2 and Table 1) in the monitored injured areas $(410 \mu \mathrm{m}$ length). However, in the vWF-deficient mice, platelet interaction with the vessel wall usually started after 1-2 minutes, and the number of early platelet depositions in the interval 3-5 minutes after $\mathrm{FeCl}_{3}$ was fivefold less than that seen in wild-type mice (Table 1). Thus, vWF is a dominant, but not the only, molecule involved in early platelet/vessel wall interactions.

By bridging the subendothelium matrix and platelet GPIb $\alpha, v W F$ contributes to the activation of the integrin $\alpha I I b \beta 3$ on the platelets through an inside-out signaling pathway (27), leading to platelet aggregation. In addition, vWF itself can bridge platelets by binding $\alpha \mathrm{IIb} \beta 3$ at high shear (28). To examine the effect of vWF deficiency in platelet aggregation, the time required for the first appearance of a significant thrombus (diameter $>20 \mu \mathrm{m}$ ) was measured. Not surprisingly, this time was about twice as long as in wild-type animals (Table 1). Nevertheless, stable thrombi were formed in the absence of vWF, and eventually multiple thrombi fused to form a larger thrombus along the injured vessel wall (Figure 2). One of the striking phenomena observed in the vWF-deficient mice was that thrombus growth often arrested, leaving an open channel through which blood flow could pass (Figure 2). About 90\% of vWFdeficient mice formed this small channel within the thrombus, which lasted at least 5 minutes (Figure 3a). These open channels prevented half of the arterioles from reaching complete occlusion and dramatically prolonged occlusion time (Figure $3 \mathrm{~b}$ ). This result indicates that vWF is a critical mediator of vascular occlusion as the diameter of the lumen is decreased by the large thrombus and the shear rate becomes very high.

Unstable thrombi and downstream vessel occlusion in Fgdeficient mice. Fg is thought to be a critical molecule involved in thrombus formation. First, it is the major ligand of $\alpha \operatorname{IIb} \beta 3$ that mediates platelet aggregation.
Second, the formation of an insoluble fibrin matrix could be important in the stabilization of the thrombus (29). We observed prominent platelet aggregation in all injured vessels in the $\mathrm{Fg}^{-/-}$mice by intravital microscopy. There was no significant difference in early platelet deposition or in the time required for thrombus initiation (Table 1). However, thrombi in these mice failed to resist shear stress when they grew to a certain size and were eventually stripped off and carried away downstream (Figures 2 and $3 \mathrm{c}$ ). Although all vessels occluded and the mean occlusion time was indistinguishable from their wild-type counterparts (Figure 3b), none occluded at the site of injury (Table 2). Thus, frequent embolization with a downstream occlusion is a major characteristic of Fgdeficient mice after vascular injury.

Thrombi formed and vessels occluded in $\mathrm{vWF} / \mathrm{Fg}^{-1-}$ mice. No human suffering from both severe vWd and afibrinogenemia has yet been reported. Interestingly, $v \mathrm{WF} / \mathrm{Fg}^{-1-}$ mice survived to adulthood, although $23 \%$ of the offspring died before weaning. In the adult animals there were no signs of anemia, and the platelet count was normal.

Surprisingly, although delayed, platelet adhesion and aggregation still occurred despite the absence of the two key adhesion molecules (Figure 2). Early platelet deposition and time required for initiation of thrombus were similar to those in $v W^{-/-}$mice (Table 1). Frequent embolization leading to downstream occlusion occurred as in $\mathrm{Fg}^{-1-}$ mice (Figure $3 \mathrm{c}$ and Table 2). However, the thrombi in the double-knockout mice were more fragile than those in either $\nu \mathrm{WF}^{-/-}$or $\mathrm{Fg}^{-/-}$mice. Dissociation and formation of small emboli were frequently observed, appearing to decrease the frequency of generation of large emboli (Figure 3c). The occlusion time in the doubly deficient mice was actually shorter than that in vWF-deficient mice (Figure 3b). This is likely due to the combination of two effects: (a) the significant numbers of small emboli formed from dissociating thrombi that blocked the intrathrombus flow channels, which may have formed due to vWF deficiency, and (b) downstream occlusion in these vessels produced by embolization, such as occurred in $\mathrm{Fg}^{-1}$ mice.

\section{Table 2}

Percentage of arterioles reaching occlusion after $\mathrm{FeCl}_{3}$ injury and the sites of the occlusion

\begin{tabular}{lcccc}
\hline & Wild-type & $\mathrm{vWF}^{-/-}$ & $\mathrm{Fg}^{-1-}$ & $\mathrm{vWF} / \mathrm{Fg}^{-/-}$ \\
$\begin{array}{l}\text { Percentage of } \\
\begin{array}{l}\text { vessels occluded } \\
\text { Percentage of vessels }\end{array}\end{array}$ & 100 & 50 & 100 & 72.7 \\
\hline
\end{tabular}

occluded at the

site of injury

The occlusion sites in wild-type and vWF-deficient mice were in the injured area. However, none of the occlusive thrombi formed in $\mathrm{Fg}^{-1-}$ mice were at the injured site $\left(\chi^{2}=28.99, P<0.005\right.$ as compared with wild-type). There was also a significant difference in the occlusion sites between $\mathrm{Fg}^{-1-}$ mice and $v W F / F^{-1-}$ mice $\left(\chi^{2}=5.19, P<0.05\right)$ because some of the doubly deficient vessels occluded in the area of injury. 


\section{Figure 2}

Characteristics of thrombus growth differ with mouse genotype. Times after $\mathrm{FeCl}_{3}$-induced injury are indicated (min). In wild-type mice (WT), numerous adherent fluorescently labeled platelets can be seen 4 minutes after $\mathrm{FeCl}_{3}$ treatment. Thrombi usually grow fast and cause vessels to occlude at the site of injury (15 minutes). In $v W F^{-/}$mice, very few platelet-vessel wall interactions were seen at the early time points, but thrombus formation occurred (13 minutes). Thrombi usually stopped growing at the later times, leaving a small channel open (arrowheads, 29 minutes) with high shear flow. The dark thrombus at this time point indicates that few new platelets were recruited, and platelet fluorescence was bleached after a long exposure to

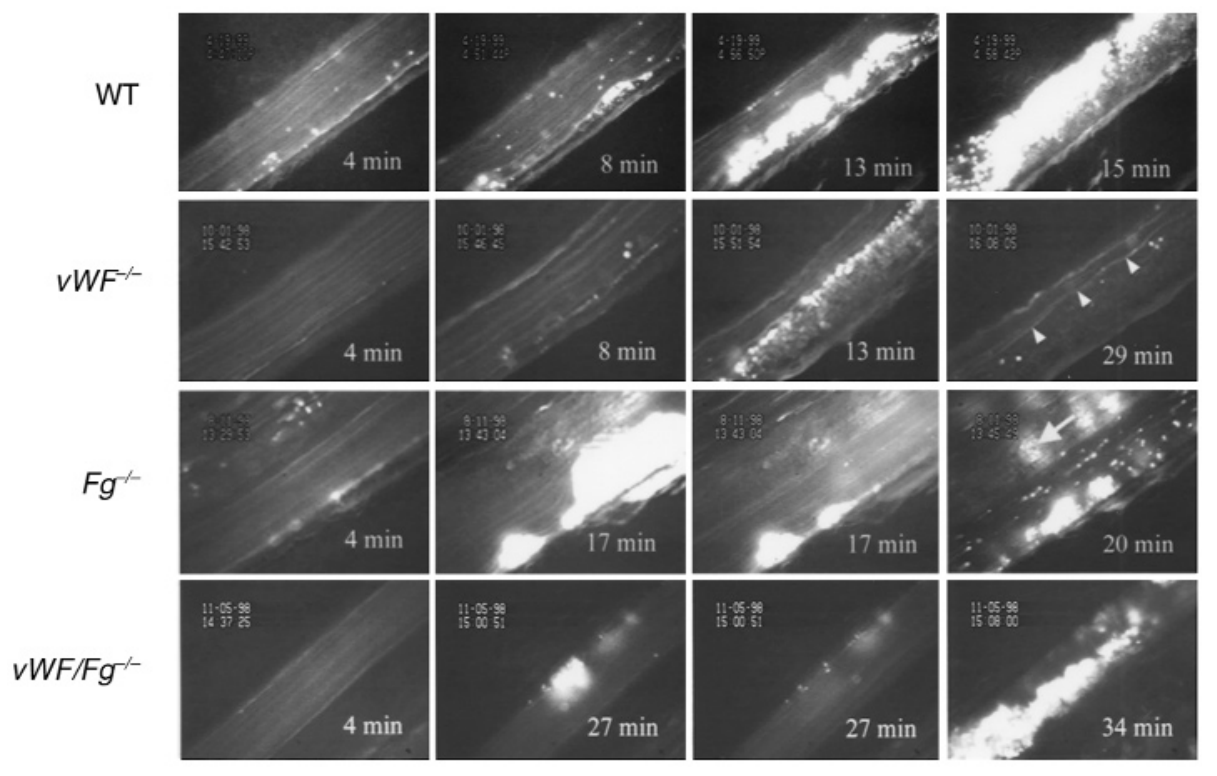
UV light. In $\mathrm{Fg}^{-1}$ mice, early platelet depositions were similar to wild-type mice (4 minutes), and thrombi grew very efficiently (bright thrombi). However, thrombi were not stable. They were often stripped off the vessel wall by blood flow (two sequential panels at 17 minutes) and ultimately led to a downstream occlusion (20 minutes with fluorescent platelets suspended in stagnant blood). The arrow in this panel ( $\mathrm{g}^{-} /{ }^{-}, 20$ minutes) shows a thrombus formed in a neighboring venule. In the double-knockout mice $\left(\mathrm{vWF}^{\left.-\mathrm{Fg}^{-1}\right)}\right.$, very few fluorescently labeled platelets were seen on the vessel wall at an early time point ( 4 minutes), but delayed thrombus formation occurred with frequent embolization (two sequential panels at 27 minutes). The majority of the double-deficient arterioles eventually occluded.

Thus, the platelet plug-formation process in the double-knockout mice presented a blend of characteristics of the single mutants.

Significant increase in platelet fibronectin in the $\mathrm{Fg}^{-1-}$ and $v \mathrm{WF} / \mathrm{Fg}^{-/-}$mice. The molecular mechanisms involved in the formation of thrombi in $v \mathrm{WF} / \mathrm{Fg}^{-/-}$mice at high shear are very intriguing. The observed aggregation is likely mediated by a $\beta 3$ integrin, since mice deficient in $\beta 3$ do not form any thrombi in this model under identical conditions (unpublished observations). It is possible that, in order to compensate for loss of vWF and Fg, the animal upregulates synthesis of another plasma ligand or platelet-adhesion receptor. Using flow cytometry, we did not detect differences in expression levels of $\alpha \mathrm{IIb} \beta 3$ and $\beta 1$ integrins on platelets among any of the mouse genotypes used in our study (not shown). Also, the concentrations of fibronectin, thrombospondin-1, and vitronectin (the potential $\beta 3$ integrin ligands) in plasma of $v \mathrm{WF} / \mathrm{Fg}^{-/-}$mice were unchanged from wild-type (not shown), indicating that the synthesis of these adhesion molecules was also not increased. Interestingly, the amounts of fibronectin in platelets of $\mathrm{Fg}^{-/-}$and $v \mathrm{WF} / \mathrm{Fg}^{-1-}$ mice were approximately three times greater than the amounts found in the platelets of wild-type or $v \mathrm{WF}^{-/-}$mice (Figure 4). This substantial increase in platelet fibronectin content in the absence of Fg was observed in five different platelet preparations. The increase was specific to fibronectin, because no changes were found in thrombospondin-1 and vitronectin levels (Figure 4). The increase in the amount of fibronectin in platelets lacking Fg was also easily seen by immunoflu- orescent staining of platelet smears, where the granularstaining pattern of fibronectin was more intense than that seen in wild-type platelets. No elevation was found in the staining intensities for thrombospondin-1 and vitronectin (not shown). Thus, the increase in level of fibronectin in the $\alpha$-granules may aid in thrombus formation in the $\mathrm{Fg}^{-/-}$and $v \mathrm{WF} / \mathrm{Fg}^{-1-}$ mice.

\section{Discussion}

The application of intravital microscopy and knockout technology allowed us to visualize and quantitate the various stages of occlusive thrombus formation in the presence and absence of the major known platelet ligands. Our observations confirmed in vivo several key facts about the functions of vWF and Fg obtained from in vitro studies over the past 30 years and also provided several new findings. We began our study by characterizing the arterial injury model in more detail than was done previously (18). The injury to endothelium occurred rapidly (Figure 1a), leading to significant denudation of the vessel in the first 5 minutes after $\mathrm{FeCl}_{3}$ application (Figure 1b). The earliest platelet-vessel wall interactions (first minute) were seen only in wild-type and Fg-deficient mice. In the following minutes, the translocating platelets began to adhere firmly to the exposed subendothelium. In the $v \mathrm{WF}^{-/-}$mice, this early platelet deposition was dramatically reduced (Table 1). Surprisingly, some 7-10 minutes after $\mathrm{FeCl}_{3}$ application, frequent platelet-adhesion events began, even in the absence of vWF, eventually leading to total occlusion in $50 \%$ of the arterioles (Table 2). Thus, contrary to prevailing views (reviewed in ref. 30) and 
a

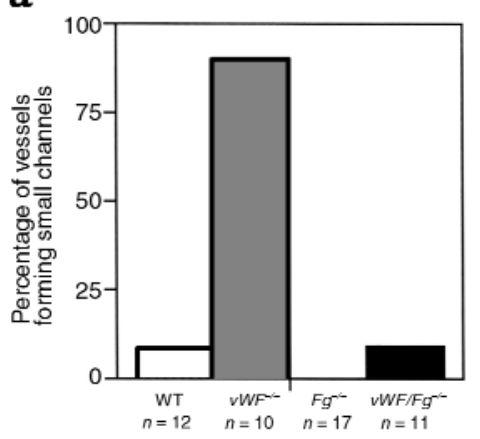

b

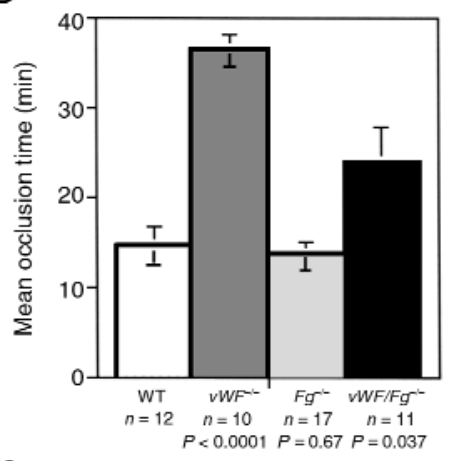

C

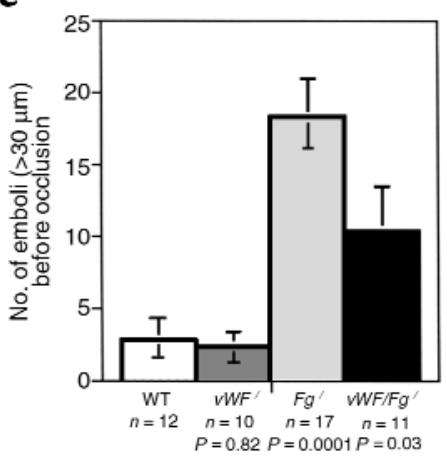

\section{Figure 3}

Quantitative analysis of thrombi formation in wild-type and mutant arterioles. (a) Frequent occurrence of narrow flow channels in thrombi of arterioles in $v W F^{-1-}$ mice. Small channels within thrombi lasting more than 5 minutes were recorded and their frequency established for each genotype. Thrombi in nine out of 10 vessels in $\mathrm{VWF}^{-/-}$mice formed these channels in the late stage, which was a frequency significantly higher than in wild-type mice $(1 / 12)\left(\chi^{2}=14.66, P<0.005\right)$ or in other genotypes of mice. (b) The effects of VWF or Fg deficiency on vessel occlusion time. The time before blood flow completely stopped in each vessel was determined. If blood flow did not cease during the 40-minute observation period, 40 minutes was used as the occlusion time. Surprisingly, the time needed to stop blood flow in $\mathrm{Fg}^{-/-}$arterioles was similar to wild-type. The occlusion time in vWF-knockout mice was longer than that of double-knockout mice $(P<0.01)$, where embolization led to a more rapid thrombus accumulation, and both were prolonged in comparison with wild-type. The $P$ values shown in this figure were determined by comparison with wild-type arterioles. (c) The roles of vWF or $\mathrm{Fg}$ in thrombus embolization. The number of large emboli (diameter $>30 \mu \mathrm{m}$ ) generated in the period before vessel occlusion was determined. Although this period was short in $\mathrm{Fg}^{-1}$ mice, the number of emboli formed in these mice was the highest. However, no statistically significant difference was found between $\mathrm{Fg}^{-1-}$ mice and double knockouts $(P=0.07)$. The $P$ values shown in this figure were determined by comparison with wild-type arterioles. even recent in vitro observations in a flow chamber (31), in our model vWF was not absolutely required to initiate platelet adhesion to subendothelium at arterial shear $\left(1300 \mathrm{~s}^{-1}\right)$ in vivo. The observed delay in platelet adhesion could be explained in two different ways: (a) deeper injury by $\mathrm{FeCl}_{3}$ was needed to expose new adhesive ligands from inner layers of the vessel wall, or (b) a blood component, which differs from $v W F$, must adhere onto the subendothelium in order to support this secondary mechanism for platelet adhesion. We cannot exclude the possibility of extracellular matrix modification by free radicals generated by $\mathrm{FeCl}_{3}$. However, in preliminary studies using a type III collagen-coated capillary tube perfused with $\nu \mathrm{WF} / \mathrm{Fg}^{-1}$ blood, we have observed platelet deposition and thrombi formation at shear of $1380 \mathrm{~s}^{-1}$ (P. Andre, H. Ni, and D.D. Wagner, unpublished observation). Thus, the platelets adhered independently of $\mathrm{FeCl}_{3}$ treatment.

The platelet receptor(s) and ligands mediating the delayed adhesion step in the absence of the vWF-GPIb pathway remain to be identified. A potential candidate is the integrin collagen-receptor $\alpha 2 \beta 1$ (10), whose ability to act in synergy with GPIb $\alpha$ and $\alpha \operatorname{IIb} \beta 3$ to promote stable platelet adhesion and activation was demonstrated (9). Indeed, patients with congenital defects in $\alpha 2 \beta 1$ have a bleeding tendency (32). Other candidates include the fibronectin receptor $\alpha 5 \beta 1$ (9), laminin receptor $\alpha 6 \beta 1$ (33), the collagen receptor GPVI (34), and thrombospondin receptors (35). Although $\alpha \mathrm{IIb} \beta 3$ may contribute to platelet-vessel wall adhesion at high shear (36), clearly adhesion exists in mice lacking both vWF and $\beta 3$ integrin (our preliminary observations).

An important observation in the vWF-deficient mice was the persistence of an open channel within or outside the thrombus occurring in a large majority of vessels (Figures 2 and 3a). Some of these channels remained stable for more than 25 minutes. The shear rate within the channel was measured in one of the animals and found to be $1,900 \mathrm{~s}^{-1}$ and $2,700 \mathrm{~s}^{-1}$ at the two extremities of the channel. It appeared that $\mathrm{vWF}$ was absolutely required for subsequent accrual of the thrombus, thus demonstrating the need for vWF in platelet-platelet firm adhesion at high shear. The presence of open channels was also observed in the hemostatic plugs formed in vWd pigs (37). Although the occlusion time in $v \mathrm{WF}^{-/-}$mice was greatly prolonged, the thrombi that formed were well anchored to the vessel wall (Figure 3c). This corroborates recent in vitro data showing that thrombus formed at low shear on collagen with blood from patients with type $3 \mathrm{vWd}$ (lacking vWF) will remain stable when shear is increased to arterial strength (31). Thus in vWd, despite the formation of well-anchored thrombi, the bleeding may continue by way of the high-shear channels.

Despite the fact that Fg is thought to be the principal ligand for $\alpha \mathrm{IIb} \beta 3$-mediated homotypic platelet adhesion $(13,38)$, the ability to form thrombi easily may be common to both afibrinogenemic mice and humans because blood from afibrinogenemic patients forms thrombi on subendothelium in a perfusion chamber 
$(31,39)$. However, the thrombi generated in the $\mathrm{Fg}^{-1}$ arterioles were highly unstable. The entire thrombus appeared to translocate slowly on the vessel wall (perhaps still moving on vWF) and embolized frequently (Figures 2 and $3 \mathrm{c}$ ), leading to a downstream vessel occlusion (Table 2). The weakest point in the Fg-deficient thrombi appeared to be located at the thrombus-vessel wall interface. This suggests that Fg or fibrin is very important in securing platelet-vessel wall adhesion. It is possible that the key role of Fg at high shear is not in platelet aggregation but rather in fibrin formation to stabilize the growing thrombus. The embolization observed in the absence of $\mathrm{Fg} /$ fibrin has important clinical implications since fatal pulmonary embolus formation has been reported in afibrinogenemic patients $(40,41)$. The bleeding problems observed in these patients are likely due to the inability to form a productive platelet plug at the site of the wound, leaving severed vessels open.

The residual platelet aggregation seen in afibrinogenemic patients is thought to be primarily mediated by vWF (42). The fact that injured vessels of mice deficient in both vWF and Fg still formed large occlusive thrombi was thus unexpected and intriguing. Our first concern upon making this observation was whether the $\mathrm{FeCl}_{3}$ could induce artifactual platelet agglutination independent of adhesion receptors and their ligands. However, this possibility was ruled out by the finding that mice deficient in $\beta 3$ integrin, lacking the $\alpha \operatorname{IIb} \beta 3$ receptor, were unable to form thrombi in the same thrombosis model. Thus, it appears that not only was the thrombus formation in the $v \mathrm{WF} / \mathrm{Fg}^{-1-}$ mice a specific phenomenon, but the molecule responsible for the platelet crosslinking was a ligand of a $\beta 3$ integrin. What are the candidates? Vitronectin was shown to bind $\alpha \operatorname{IIb} \beta 3$ on stimulated platelets (43). The platelet $\alpha$-granule vitronectin is multimeric (44) and thus could be capable of platelet crosslinking. But the absence of vitronectin was recently shown to accelerate thrombus formation in an in vivo thrombosis model of carotid artery injury (24), making vitronectin less likely to promote thrombus growth in our model. A second candidate is thrombospondin-1, which contains three arginine-glycine-aspartic acid sequences (45). Although its plasma concentration is negligible, it is located in the $\alpha$-granules, and its concentration after degranulation could be sufficient to support platelet aggregation. In addition, thrombospondin1-derived peptides were reported to inhibit the secretiondependent phase of platelet aggregation (46). The absence of Fg or vWF did not affect the $\alpha$-granule content of any of these proteins. In contrast, the platelet level of fibronectin, a third known ligand for $\alpha \operatorname{IIb} \beta 3$ (47), was increased threefold (Figure 4), making the granular-staining pattern of the platelets visibly brighter (not shown). This observation makes it likely that fibronectin plays an active role in the biology of the platelet.

The role of fibronectin in platelet aggregation is controversial. A $\mathrm{mAb}$ to fibronectin was shown to interfere with thrombin-induced platelet aggregation (48).
However, in different studies plasma fibronectin was shown to either inhibit, or have no effect, or promote platelet aggregation (49). The increase in $\alpha$-granular fibronectin could be due either to a compensatory rise in biosynthesis or increased uptake of fibronectin from plasma. We think that the increase in synthesis is less likely because the level of plasma fibronectin was unchanged, and thus any increase would have to be megakaryocyte specific. It is more likely that in the absence of Fg, the $\alpha \operatorname{IIb} \beta 3$, which normally imports Fg into the platelet $\alpha$-granules (50), now imports fibronectin. This $\alpha$ IIb $\beta 3$-bound fibronectin would now be available for platelet aggregation. For the moment, we do not have any evidence that fibronectin actually crosslinks the platelets. However, it appears likely that increased platelet fibronectin can compensate in part for the loss of $\mathrm{Fg}$. It is harder to determine how significant a role fibronectin may play in normal hemostasis when Fg and vWF are present. Indeed, if fibronectin or another $\alpha \operatorname{IIb} \beta 3$ ligand is active in wildtype animals, one might expect that the absence of such a ligand would cause a known bleeding disorder. Neither vitronectin nor thrombospondin-1 has been linked to a bleeding diathesis, and the corresponding deficient mice do not have a bleeding phenotype. We cannot determine what effect fibronectin deficiency would have on hemostasis, since fibronectin deficiency causes early embryonic lethality in mice (51) and, presumably, humans.

We hope that further experiments using platelets and plasma from the $v W F / \mathrm{Fg}^{-1-}$ mice will help to identify conclusively the additional molecular players involved in platelet adhesion and aggregation uncovered by the present study.

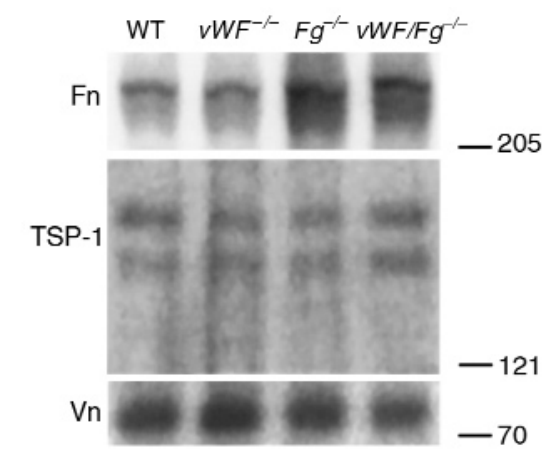

\section{Figure 4}

Increased fibronectin content in platelets of $\mathrm{Fg}^{-1}$ and $\mathrm{vWF} / \mathrm{Fg}^{-1-}$ mice. For each genotype, platelets were isolated from pooled blood of six mice. Gel-filtered platelets $\left(2 \times 10^{7}\right)$ were analyzed by Western blot. The position of migration of molecular mass standards is shown on the right (in kiloDaltons). Platelet vitronectin $(\mathrm{Vn})$ and thrombospondin-1 (TSP-1) levels were the same in all samples. In contrast, the amount of fibronectin in platelets from either the $\mathrm{Fg}^{-1-}$ or $v W F / \mathrm{Fg}^{-1}$ mice was three times greater than from the wild-type mice or $v W F^{-1-}$ mice. The doublet seen with anti-thrombospondin1 is a consequence of proteolytic cleavage of thrombospondin-1. 


\section{Acknowledgments}

We thank Paul Frenette and Patrick Andre for their advice, Maria Economopoulos for $v \mathrm{WF}^{-/-}$mouse husbandry, Simin Saffaripour and Faisal Mahmood Khan for assistance in immunohistochemical staining, Jane Trevithick and Irina Knezevic for help with Western blots, and Lesley Cowan for assistance preparing the manuscript. This work was supported in part by NIH grants R37 HL-41002 (D.D. Wagner), R01 HL-47826 (J.L. Degen), and P01 HL-414484 (R.O. Hynes). H. Ni is a fellow of the Heart and Stroke Foundation of Canada. T.N. Sato is an Established Investigator of the American Heart Association. R.O. Hynes is an Investigator of Howard Hughes Medical Institute.

1. von Willebrand, E. 1926. Hereditar psseudohemofili. Fin. Lakaresallsk. Handl. 67:87-112.

2. Gugler, E., and Lüscher, E.F. 1965. Platelet function in congenital afibrinogenemia. Thromb. Diath. Haemorrh. 14:361-373.

3. Ewenstein, B.M. 1997. von Willebrand's disease. Annu. Rev. Med. 48:525-542.

4. Martinez, J. 1997. Congenital dysfibrinogenemia. Curr. Opin. Hematol. 4:357-365.

5. Tschopp, T.B., Weiss, H.J., and Baumgartner, H.R. 1974. Decreased adhesion of platelets to subendothelium in von Willebrand's disease.J. Lab. Clin. Med. 83:296-300

6. Sakariassen, K.S., Bolhuis, P.A., and Sixma, J.J. 1979. Human blood platelet adhesion to artery subendothelium is mediated by factor VIII-Von Willebrand factor bound to the subendothelium. Nature. 279:636-638.

7. De Marco, L., Girolami, A., Russell, S., and Ruggeri, Z.M. 1985. Interaction of asialo von Willebrand factor with glycoprotein Ib induces fibrinogen binding to the glycoprotein IIb/IIIa complex and mediates platelet aggregation. J. Clin. Invest. 75:1198-1203.

8. Savage, B., Saldiver, E., and Ruggeri, Z.M. 1996. Initiation of platelet adhesion by arrest onto fibrinogen or translocation on von Willebrand factor. Cell. 84:289-297.

9. Savage, B., Almus-Jacobs, F., and Ruggeri, Z.M. 1998. Specific synergy of multiple substrate-receptor interactions in platelet thrombus formation under flow. Cell. 94:657-666.

10. Nieuwenhuis, H.K., Sakariasssen, K.S., Houdijk, W.P.M., Nievelstein, P.F.E.M., and Sixma, J.J. 1986. Deficiency of platelet membrane glycoprotein Ia associated with a decreased platelet adhesion to subendothelium: a defect in platelet spreading. Blood. 68:692-695.

11. Sakariassen, K.S., Nievelstein, P.F.E.M., Coller, B.S., and Sixma, J.J. 1986. The role of platelet membrane glycoproteins Ib and IIIb-IIIa in platelet adherence to human artery subendothelium. Br. J. Haematol. 63:681-691.

12. Mustard, J.F., Packham, M.A., Kinlough-Rathbone, R.L., Perry, D.W., and Regoeczi, E. 1978. Fibrinogen and ADP-induced platelet aggregation. Blood. 52:453-466.

13. Coller, B.S. 1980. Interaction of normal, thrombasthenic, and BernardSoulier platelets with immobilized fibrinogen: defective platelet-fibrinogen interaction in thrombasthenia. Blood. 55:169-178.

14. Turitto, V.T., Weiss, H.J., and Baumgartner, H.R. 1984. Platelet interaction with rabbit subendothelium in von Willebrand's disease: altered thrombus formation distinct from defective platelet adhesion. J. Clin. Invest. 74:1730-1741.

15. Phillips, D.R., Charo, I.F., and Scarborough, R.M. 1991. GPIIb-IIIa: the responsive integrin. Cell. 65:359-362.

16. Ruggeri, Z.M., Dent, J.A., and Saldivar, E. 1999. Contribution of distinct adhesive interactions to platelet aggregation in flowing blood. Blood. 94:172-178

17. Nachman, R.L., and Leung, L.L. 1982. Complex formation of platelet membrane glycoproteins IIb and IIIa with fibrinogen. J. Clin. Invest. 69:263-269.

18. Denis, C., et al. 1998. A mouse model of severe von Willebrand disease: defects in hemostasis and thrombosis. Proc. Natl. Acad. Sci. USA. 95:9524-9529.

19. Suh, T.T., et al. 1995. Resolution of spontaneous bleeding events but failure of pregnancy in fibrinogen-deficient mice. Genes Dev. 9:2030-2033.

20. Kurz, K.D., Main, B.W., and Sandusky, G.E. 1990. Rat model of arterial thrombosis induced by ferric chloride. Thromb. Res. 60:269-280

21. Schlaeger, T.M., et al. 1997. Uniform vascular-endothelial-cell-specific gene expression in both embryonic and adult transgenic mice. Proc. Natl. Acad. Sci. USA. 94:3058-3063.

22. Mautner, V., and Hynes, R.O. 1977. Surface distribution of LETS protein in relation to the cytoskeleton of normal and transformed cells. J. Cell Biol. 75:743-768.

23. Marsh Lyle, E., et al. 1998. Assessment of thrombin inhibitor efficacy in a novel rabbit model of simultaneous arterial and venous thrombosis. Thromb. Haemost. 79:656-662.

24. Fay, W.P., Parker, A.C., Ansari, M.N., Zheng, X., and Ginsburg, D. 1999. Vitronectin inhibits the thrombotic response to arterial injury in mice. Blood. 93:1825-1830.

25. Wagner, D.D., Olmsted, J.B., and Marder, V.J. 1982. Immunolocalization of von Willebrand protein in Weibel-Palade bodies of human endothelial cells. Cell Biol. 95:355-360.

26. Tsakiris, D.A., Scudder, L., Hodivala-Dilke, K., Hynes, R.O., and Coller, B.S. 1999. Hemostasis in the mouse (Mus musculus): a review. Thromb. Haemost. 81:177-188.

27. Shattil, S.J., Kashiwagi, H., and Pampori, N. 1998. Integrin signaling: the platelet paradigm. Blood. 91:2645-2657.

28. Goto, S., Ikeda, Y., Saldivar, E., and Ruggeri, Z.M. 1998. Distinct mechanisms of platelet aggregation as a consequence of different shearing flow conditions. J. Clin. Invest. 101:479-486.

29. Berndt, M.C., et al. 1995. The molecular mechanism of platelet adhesion. Aust. N. Z. J. Med. 25:822-830.

30. Ruggeri, Z.M. 1997. Mechanisms initiating platelet thrombus formation. Thromb. Haemost. 78:611-616.

31. Tsuji, S., et al. 1999. Real-time analysis of mural thrombus formation in various platelet aggregation disorders: distinct shear-dependent roles of platelet receptors and adhesive proteins under flow. Blood. 94:968-973.

32. Nieuwenhuis, H.K., Akkerman, J.W.N., Houdijk, W.P.M., and Sixma, J.J. 1985. Human blood platelets showing no response to collagen fail to express surface glycoprotein Ia. Nature. 318:470-472.

33. Sonnenberg, A., Modderman, P.W., and Hogervorst, F. 1988. Laminin receptor on platelets is the integrin VLA-6. Nature. 336:487-489.

34. Moroi, M., et al. 1996. Analysis of platelet adhesion to a collagen-coated surface under flow conditions: the involvement of glycoprotein VI in the platelet adhesion. Blood. 88:2081-2092.

35. Agbanyo, F.R., Sixma, J.J., de Groot, P.G., Languino, L.R., and Plow, E.F. 1993. Thrombospondin-platelet interactions. Role of divalent cations, wall shear rate, and platelet membrane glycoproteins. J. Clin. Invest. 92:288-296.

36. Weiss, H.J., et al. 1989. Fibrinogen-independent platelet adhesion and thrombus formation on subendothelium mediated by glycoprotein IIb-IIIa at high shear rate. J. Clin. Invest. 83:288-297.

37. Sawada, Y., Fass, D.N., Katzmann, J.A., Bahn, R.C., and Bowie, E.J. 1986. Hemostatic plug formation in normal and von Willebrand pigs: the effect of the administration of cryoprecipitate and a monoclonal antibody to von Willebrand factor. Blood. 67:1229-1239.

38. Frojmovic, M.M. 1998. Platelet aggregation in flow: differential roles for adhesive receptors and ligands. Am. Heart J. 135(Suppl.):S119-S131.

39. Weiss, H.J., Turitto, V.T., Vicic, W.J., and Baumgartner, H.R. 1984. Fibrin formation, fibrinopeptide A release, and platelet thrombus dimensions on subendothelium exposed to flowing native blood: greater in factor XII and XI than in factor VIII and IX. Blood. 63:1004-1014.

40. Ingram, G.I., McBrien, D.J., and Spencer, H. 1966. Fatal pulmonary embolus in congenital fibrinopenia. Report of two cases. Acta Haematol. 35:56-62.

41. Cronin, C., Fitzpatrick, D., and Temperley, I. 1988. Multiple pulmonary emboli in a patient with afibrinogenaemia. Acta Haematol. 7:53-54

42. DeMarco, L., Girolami, A., Zimmerman, T.S., and Ruggeri, Z.M. 1986. von Willebrand factor interaction with the glycoprotein IIb/IIa complex. Its role in platelet function as demonstrated in patients with congenital afibrinogenemia. J. Clin. Invest. 77:1272-1277.

43. Thiagarajan, P., and Kelly, K.L. 1988. Exposure of binding sites for vitronectin on platelets following stimulation. J. Biol. Chem. 263:3035-3038.

44. Seiffert, D., and Schleef, R.R. 1996. Two functionally distinct pools of vitronectin $(\mathrm{Vn})$ in the blood circulation: identification of a heparin-binding competent population of $\mathrm{Vn}$ within platelet alpha-granules. Blood. 88:552-560.

45. Lawler, J., and Hynes, R.O. 1986. The structure of thrombospondin, an adhesive glycoprotein with multiple calcium-binding sites and homologies with several different proteins. J. Cell. Biol. 103:1635-1648.

46. Rabhi-Sabile, S., Thibert, V., and Legrand, C. 1996. Thrombospondin peptides inhibit the secretion-dependent phase of platelet aggregation. Blood Coagul. Fibrinolysis. 7:237-240.

47. Plow, E.F., and Ginsberg, M.H. 1981. Specific and saturable binding of plasma fibronectin to thrombin-stimulated human platelets. J. Biol. Chem. 246:9477-9482.

48. Dixit, V.M., et al. 1985. Inhibition of platelet aggregation by a monoclonal antibody against human fibronectin. Proc. Natl. Acad. Sci. USA. 82:3844-3848.

49. Hynes, R.O. 1990. Fibronectins. Springer Verlag. New York, New York, USA. $546 \mathrm{pp}$

50. Handagama, P., Scarborough, R.M., Shuman, M.A., and Bainton, D.F. 1993. Endocytosis of fibrinogen into megakaryocytes and platelet $\alpha$-granules is mediated by $\alpha I I b \beta 3$ (glycoprotein IIb-IIIa). Blood. 82:135-138.

51. George, E.L., Georges-Labouesse, E.N., Patel-King, R.S., Rayburn, H., and Hynes, R.O. 1993. Defects in mesoderm, neural tube and vascular development in mouse embryos lacking fibronectin. Development. 119:1079-1091. 